\title{
Re: "Transcatheter treatment for refractory angina with the coronary sinus Reducer" by Maayan Konigstein et al
}

\author{
Yoav $\mathrm{Paz}^{1 *}$, MD, MHA; Amihay Shinfeld², MD
}

\author{
1. General Intensive Care Unit, Tel Aviv Medical Center, Tel Aviv, Israel, and the Sackler Faculty of Medicine, Tel Aviv University, \\ Tel Aviv, Israel; 2. Department of Cardiac Surgery, Chaim Sheba Medical Center, Tel Hashomer, Tel Aviv, Israel, and the Sackler \\ Faculty of Medicine, Tel Aviv University, Tel Aviv, Israel
}

We read with interest the paper by Konigstein et $\mathrm{al}^{1}$, and wish to commend the authors for their significant contribution.

In the discussion section the authors suggest that the antiischaemic effect of the Coronary Sinus Reducer ${ }^{\mathrm{TM}}$ Stent (CSRS) (Neovasc Medical, Inc., Or Yehuda, Israel) is based on the hypothesis described by Camici et $\mathrm{al}^{2}$. We would like to point out that, while Camici et al published this hypothesis in the New England Journal of Medicine in 2007, the initial experiments with the CSRS were performed a decade before. Even Banai et al had used the CSRS before Camici et al published their hypothesis, when they implemented the first-in-man study using this stent ${ }^{3}$.

Konigstein et al $^{1}$ support their rationalisation of the anti-ischaemic effect of the CSRS by quoting Ido et $\mathrm{al}^{4}$, a study which examined the effects of coronary sinus occlusion on coronary collateral blood flow and on the distribution of regional myocardial blood flow in dogs. We wish to emphasise that there is a substantial difference between human and dog morphology of veins that drain into the coronary sinus ${ }^{5}$.

In the mid 1990s we tested a new strategy supporting the ischaemic myocardium. This strategy included: 1) catheterisation of the coronary venous system rather than catheterisation of the coronary arteries, and 2) reduction of the coronary sinus (CS) effective cross area, as opposed to the expansion of a narrowed coronary artery. At that time, the main concept behind this strategy was to rebuild retrograde coronary pressure that would be attenuated by the atherosclerotic disease. In order to test this strategy, we designed and manufactured the first CSRS.

In a preliminary non-ischaemic pig model we succeeded in increasing the mean CS pressure from 7.0 to $24.6 \mathrm{mmHg}(\mathrm{p}=0.011)$ after CSRS deployment. Further studies in a non-ischaemic pig model were devoted to macroscopic and histological investigations of the treated hearts, in particular investigating whether any structural or histological damage, such as an infarct, had occurred after CSRS implantation.

While looking for such damage, these studies revealed that eight to 12 weeks of coronary sinus narrowing produced macroscopic epicardial and intramyocardial new blood vessels - neovascularisation. Histopathological analysis described these findings as follows: significant proliferation of small to medium-sized vessels, containing smooth muscle representing coronary collaterals. This was evident in almost all specimens, representing various myocardial anatomical areas, including specimens from the anterior and mid-posterior wall. According to these unpredicted neovascularisation findings after CSRS implantation in a non-ischaemic pig model, we created the name "Neovasc" for this novel CSRS device.

Backwards pressure elevation in the venules and capillaries starts immediately after CSRS deployment and a few weeks before clinical improvement. However, neovascularisation created by CSRS a few weeks following deployment seems to be the main contributing factor for the anti-ischaemic effect, and correlates well with the report by Konigstein et al, in which clinical improvement was, in most cases, reported to have started a few weeks following the procedure.

\section{Conflict of interest statement}

Y. Paz and A. Shinfeld are the inventors of the Neovasc Coronary Sinus Reducer Stent. They currently have no commercial or other association with the company whose product is the subject of this letter, or with a company that manufactures comparable products.

\section{References}

1. Konigstein M, Meyten N, Verheye S, Schwartz M, Banai S. Transcatheter treatment for refractory angina with the coronary sinus Reducer. EuroIntervention. 2014;9:1158-64.

2. Camici PG, Crea F. Coronary microvascular dysfunction. N Engl J Med. 2007;356:830-40.

3. Banai S, Ben Muvhar S, Parikh KH, Medina A, Sievert H, Seth A, Tsehori J, Paz Y, Sheinfeld A, Keren G. Coronary sinus reducer stent for the treatment of chronic refractory angina pectoris: a prospective, open-label, multicenter, safety feasibility first-inman study. J Am Coll Cardiol. 2007;49:1783-9.

4. Ido A, Hasebe N, Matsuhashi H, Kikuchi K. Coronary sinus occlusion enhances coronary collateral flow and reduces subendocardial ischemia. Am J Physiol Heart Circ Physiol. 2001;280: H1361-7.

5. Esperanca Pina JA, Coreia M, O’Neill JG, Rendas AB. Morphology of the veins draining the coronary sinus of the dog. Acta Anat (Basel). 1981;109:122-8.

*Corresponding author: General Intensive Care Unit, Tel Aviv Medical Center, Tel Aviv, Israel.

E-mail:pazyoav@bezeqint.net 


\title{
The Coronary Sinus Reducer improves angina and ischaemia by redistribution of blood from non-ischaemic to ischaemic myocardium
}

\author{
Maayan Konigstein ${ }^{1}$, MD; Nathalie Meyten², MD; Stefan Verheye², MD; Marc Schwartz ${ }^{3}$, RCIS; \\ Shmuel Banai ${ }^{*}$, MD

\section{Department of Cardiology, Tel Aviv Medical Center, Tel Aviv, Israel, and the Sackler Faculty of Medicine, Tel Aviv University, Tel Aviv, Israel; 2. Antwerp Cardiovascular Center, ZNA Middelheim, Antwerp, Belgium; 3. Neovasc, Inc., Richmond, BC, Canada}

Drs Paz and Shinfeld argue that the anti-ischaemic mechanism of action of the coronary sinus Reducer is by promoting neovascularisation. The scientific basis of their theory is a series of preclinical experiments which they say they performed in the mid 1990s.

As far as I comprehend the field of myocardial neovascularisation and myocardial angiogenesis ${ }^{6-10}$, pressure gradients that are built between blood vessels within the myocardium (as in the case of the Reducer) are responsible for the opening of preformed collaterals and not for the creation of new blood vessels (neovascularisation).

Since the Reducer is implanted in patients with chronic refractory angina, chronic myocardial ischaemia is present long before the Reducer is implanted. The chronic ischaemia activates angiogenic processes, generating neovessels, mainly capillaries, small arterioles, and venules ${ }^{11}$. Therefore, the ischaemia-induced myocardial neovascularisation process exerts itself long before the Reducer is implanted.

After implantation, the Reducer does not cause any pressure gradient until four to six weeks after implantation, when the metal mesh is covered with tissue. When pressure gradient is built, the changes in vascular tone provide a quick functional adaptation to accommodate rapid changes in metabolic demand by the development of existing collateral vessels, and by rapid changes in vessel diameter and drop in resistance to flow in the subendocardial myocardium ${ }^{11}$.

As we state in our manuscript ${ }^{12}$, the beneficial effects of the Reducer revolve around the consequence of ischaemia-induced impaired contractility and elevated left ventricular end-diastolic pressure (LVEDP). Elevated LVEDP exerts an external pressure on the subendocardial capillaries and arterioles which increases the resistance to flow in the subendocardium, and worsens subendocardial ischaemia. Heightened CS pressure causes backwards pressure elevation in the venules and capillaries which will result in a dilatation of the capillaries' and arterioles' diameter and a reduction in resistance to flow to the subendocardium. Consequently, enhancement of blood flow to the ischaemic subendocardial layers will occur, with improved contractility and reduced LVEDP which will further reduce subendocardial resistance.
Nevertheless, if Drs Paz and Shinfeld have proven otherwise, they should share the results of their scientific work with the readers of EuroIntervention, and they should cite their peer-reviewed scientific publications on the subject, so we can all read and learn.

\section{Conflict of interest statement}

S. Banai is the Medical Director of Neovasc Inc, Vancouver, Canada. M. Schwartz is the Director of Clinical Affairs and an employee of Neovasc Inc. The other authors have no conflicts of interest to declare.

\section{References}

6. Banai S, Jaklitsch MT, Casscells W, Shou M, Shrivastav S, Correa R, Epstein SE, Unger EF. Effects of acidic fibroblast growth factor on normal and ischemic myocardium. Circ Res. 1991;69:76-85.

7. Unger EF, Banai S, Shou M, Jaklitsch M, Jaye M, Epstein SE. A model to assess interventions to improve collateral blood flow: continuous administration of agents into the left coronary artery of dogs. Cardiovasc Res. 1993;27:785-91.

8. Unger EF, Banai S, Shou M, Lazarous D, Jaklitsch MT, Scheinowitz M, Correa R, Klingbeil C, Epstein SE. Basic fibroblast growth factor enhances myocardial collateral flow in a canine model. Am J Physiol. 1994;266:H1588-95.

9. Banai S, Jaklitsch MT, Shou M, Lazarous D, Scheinowitz M, Biro S, Epstein SE, Unger EF. Angiogenic-induced enhancement of collateral blood flow to ischemic myocardium by vascular endothelial growth factor in dogs. Circulation. 1994;89:2183-9.

10. Banai S, Shweiki D, Pinson A, Chandra M, Lazarovici G, Keshet E. Upregulation of vascular endothelial growth factor expression induced by myocardial ischemia: implications for coronary angiogenesis. Cardiovasc Res. 1994;28:1176-9.

11. Lehoux S, Lévy BI. Collateral artery growth - making the most of what you have. Circ Res. 2006;99:567-9.

12. Konigstein M, Meyten N, Verheye S, Schwartz M, Banai S. Transcatheter treatment for refractory angina with the coronary sinus Reducer. EuroIntervention. 2014;9:1158-64.

*Corresponding author: Interventional Cardiology Department, Tel Aviv Medical Center, 6 Weizman Street, Tel Aviv, 64239, Israel.E-mail: banais@netvision.net.il 